\title{
GENERALIZED TRANSVERSELY PROJECTIVE STRUCTURE ON A TRANSVERSELY HOLOMORPHIC FOLIATION
}

\author{
INDRANIL BISWAS
}

Received 29 November 2000 and in revised form 4 June 2001

The results of Biswas (2000) are extended to the situation of transversely projective foliations. In particular, it is shown that a transversely holomorphic foliation defined using everywhere locally nondegenerate maps to a projective space $\mathbb{C P}^{n}$, and whose transition functions are given by automorphisms of the projective space, has a canonical transversely projective structure. Such a foliation is also associated with a transversely holomorphic section of $N^{\otimes-k}$ for each $k \in[3, n+1]$, where $N$ is the normal bundle to the foliation. These transversely holomorphic sections are also flat with respect to the Bott partial connection.

2000 Mathematics Subject Classification: 37F75, 32H02, 51A45.

1. Introduction. A projective structure on a Riemann surface $X$ is defined by giving a covering of $X$ by holomorphic coordinate charts such that all the transition functions are restrictions of Möbius transformations. It is well known that the notion of a projective structure can be extended to the situation of foliations (cf. [10]). To define this generalization, let $\mathscr{F}$ be a foliation of codimension two on a real manifold $M$. Let $\left\{U_{i}\right\}_{i \in I}$ be an open covering of $M$, and let $\phi_{i}: U_{i} \rightarrow \mathbb{C}$ be submersions onto the image such that the fibers of $\phi_{i}$ are leaves for $\mathscr{F}$. A transversely projective structure on $\mathscr{F}$ is defined by imposing the condition that, for every $i, j \in I$, there is a commutative diagram

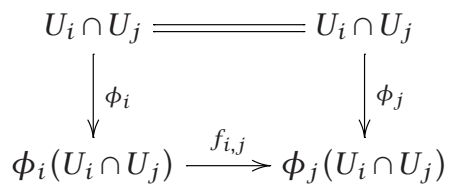

such that $f_{i, j}$ is a restriction of some Möbius transformation [10].

A holomorphic immersion $\gamma: X \rightarrow \mathbb{C P}^{n}$ of a Riemann surface $X$ is called everywhere locally nondegenerate if for every $x \in X$, the order of contact of the image $\gamma(U)$ at $\gamma(x)$, where $U$ is a neighborhood of $x$ in $X$, with any hyperplane in $\mathbb{C P}^{n}$ passing through $\gamma(x)$ is at most $n-1$ (see [3, 9]). Two such immersions are called equivalent if they differ by an automorphism of $\mathbb{C} \mathbb{P}^{n}$. A $\mathbb{C P}^{n}$-structure on $X$ is an equivalence class of an everywhere locally nondegenerate equivariant map of the universal cover of $X$ into $\mathbb{C P}^{n}$. A $\mathbb{C P}^{1}$-structure on $X$ is the same as a projective structure on $X$.

If $f: X \rightarrow \mathbb{C P}^{n}$ is a holomorphic map such that the image of $f$ is not contained in any hyperplane of $\mathbb{C} \mathbb{P}^{n}$, then there is a finite subset $S \subset X$ such that the restriction of 
$f$ to the complement $X \backslash S$ defines a $\mathbb{C P}^{n}$-structure on $X \backslash S$. Any Riemann surface has many $\mathbb{C P}^{n}$-structures. In [3], it has been shown that the space of $\mathbb{C P}^{n}$-structures on $X$, where $n \geq 2$, is canonically identified with the Cartesian product of the space of all projective structures on $X$ with the direct sum $\bigoplus_{i=3}^{n+1} H^{0}\left(X, K_{X}^{\otimes i}\right)$.

The notion of a $\mathbb{C P}^{n}$-structure can be extended to the situation of foliations which will be called a transversely $\mathbb{C P}^{n}$-structure; see Definition 2.3 for the definition of a transversely $\mathbb{C P}^{n}$-structure.

Let $\mathscr{F}$ be a transversely holomorphic foliation of complex codimension one. So the normal bundle $N$ is a transversely holomorphic line bundle. The normal bundle $N$ is equipped with the Bott partial connection obtained from the Lie bracket operation of vector fields. The transversely holomorphic structure of $N$ is compatible with the Bott partial connection.

We prove that, giving a transversely $\mathbb{C}^{n}$-structure on $\mathscr{F}_{F}$ is equivalent to giving a transversely projective structure on $\mathscr{F}$ together with a transversely holomorphic section $\omega_{k}$ of $N^{\otimes-k}$, for each $k \in[3, n+1]$, such that $\omega_{k}$ is flat with respect to the Bott partial connection (see Theorem 2.4). In particular, setting all $\omega_{k}$ to be zero we conclude that, for any transversely $\mathbb{C P}^{n}$-structure on $\mathscr{F}$ there is a canonically associated transversely projective structure on $\mathscr{F}$. When the foliation is trivial, that is, $\mathscr{F}=0$, then Theorem 2.4 is the main result of [3] (see [3, Theorem 5.5]).

It is not easy to directly construct a transversely $\mathbb{C}^{n}$-structure on a holomorphic foliation. In fact, when the foliation is trivial, namely we have a Riemann surface $X$, it is not easy to construct a map of the universal cover of $X$ to $\mathbb{C} \mathbb{P}^{n}$, which is everywhere locally nondegenerate. However, using Theorem 2.4 we can indirectly construct many examples of transversely $\mathbb{C P}^{n}$-structures, just as using [3, Theorem 5.5], we can indirectly construct examples of everywhere locally nondegenerate maps of the universal cover of a Riemann surface to $\mathbb{C P}^{n}$.

2. Transversely projective foliations defined by maps to a projective space. Let $M$ be a connected smooth real manifold of dimension $d+2$. Let $\mathscr{F}$ be a $C^{\infty}$-subbundle of rank $d$ of the tangent bundle $T M$.

DEFINITION 2.1. A transversely holomorphic structure on $\mathscr{F}$ is defined by giving the following data (see [5]):

(1) a covering of $M$ by open subsets $U_{i}$, where $i$ runs over an index set $I$. So we have $\bigcup_{i \in I} U_{i}=M$;

(2) for each $i \in I$, a submersion $\phi_{i}$ of $U_{i}$ to an open subset $D_{i}$ of $\mathbb{C}$. The restriction $\left.\mathscr{F}\right|_{U_{i}}$ is the kernel of the differential map $d \phi_{i}: T U_{i} \rightarrow \phi_{i}^{*} T D_{i}$;

(3) for every pair $i, j \in I$, there is a commutative diagram of maps

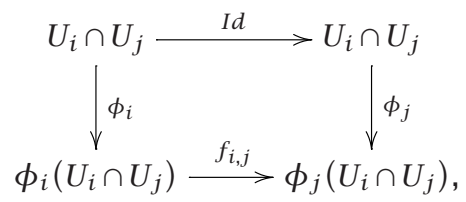

where $f_{i, j}$ is a holomorphic map. 
Two such data $\left\{U_{i}, \phi_{i}\right\}_{i \in I}$ and $\left\{U_{i}, \phi_{i}\right\}_{i \in J}$ are called equivalent if their union, namely

$$
\left\{U_{i}, \phi_{i}\right\}_{i \in I \cup J},
$$

also satisfies the above conditions. A transversely holomorphic structure on $\mathscr{F}$ will mean an equivalence class of data of the above type satisfying the three conditions.

Next we recall the definition of a transversely projective foliation.

DEFINITION 2.2. A transversely projective structure on $\mathscr{F}$ is defined by giving a data $\left\{U_{i}, \phi_{i}\right\}_{i \in I}$ exactly as in Definition 2.1, but satisfying the extra condition (apart from the three conditions) that the holomorphic maps $f_{i, j}$ in condition (3) are of the form $z \mapsto(a z+b) /(c z+d)$, where $a, b, c, d \in \mathbb{C}$ are constant scalars and $a d-b c=1$, that is, each $f_{i, j}$ is the restriction of some Möbius transformation; the scalars $a, b, c, d$ may depend on the index $i$. As before, two such data $\left\{U_{i}, \phi_{i}\right\}_{i \in I}$ and $\left\{U_{i}, \phi_{i}\right\}_{i \in J}$ are called equivalent if their union $\left\{U_{i}, \phi_{i}\right\}_{i \in I \cup J}$ is also a data for a transversely projective structure. A transversely projective structure on $\mathscr{F}$ will mean an equivalence class of such data.

Clearly, a transversely projective structure on $\mathscr{F}$ defines a transversely holomorphic structure on $\mathscr{F}$. If $\overline{\mathscr{F}}$ is a transversely holomorphic structure on $\mathscr{F}$, then a transversely projective structure on $\overline{\mathscr{F}}$ is a transversely projective structure on $\mathscr{F}_{F}$ such that, the transversely holomorphic structure defined by it coincides with $\overline{\mathscr{F}}$.

We now recall the notion of a locally nondegenerate immersion of a Riemann surface into a projective space (see [3, 9]).

Let $X$ be a Riemann surface, that is, a complex manifold of complex dimension one. Let $\mathbb{C P}^{n}, n \geq 1$, denote the $n$-dimensional projective space consisting of all lines in $\mathbb{C}^{n+1}$. A holomorphic immersion

$$
\gamma: X \rightarrow \mathbb{C P}^{n}
$$

is called everywhere locally nondegenerate if for every $x \in X$, the order of contact of the image $\gamma(U)$, where $U$ is a neighborhood of $x$ in $X$, at $\gamma(x)$ with any hyperplane in $\mathbb{C} \mathbb{P}^{n}$ passing through $\gamma(x)$ is at most $n-1$. We need to consider a neighborhood in the definition since $\gamma$ may not be injective.

An alternative description of the above nondegeneracy condition following [9] is given below.

Let

$$
0 \rightarrow S \rightarrow V \stackrel{q}{\longrightarrow} Q \longrightarrow 0
$$

be the universal exact sequence over $\mathbb{C P}^{n}$. The vector bundle $V$ is the trivial vector bundle with $\mathbb{C}^{n+1}$ as fiber and $S$ is the tautological line bundle $\mathscr{O}_{\mathbb{C P} n}(-1)$. Consider the differential

$$
d \gamma: T_{X} \longrightarrow \gamma^{*} T_{\mathbb{C P} n}=\gamma^{*} \operatorname{Hom}(S, Q)
$$

of the immersion $\gamma$; here $T_{X}$ is the holomorphic tangent bundle of $X$. Since $\gamma$ is an immersion, the homomorphism $d \gamma$ is injective. 
Now, the homomorphism $d \gamma$ gives a homomorphism

$$
\overline{d \gamma}: T_{X}^{*} \otimes \gamma^{*} S \longrightarrow \gamma^{*} Q,
$$

where $T_{X}^{*}$ is the holomorphic cotangent bundle of $X$. Let $S_{1}$ denote the inverse image $q^{-1}$ (image $(\overline{d y})$ ), where the homomorphism $q$ is defined in (2.4). The subbundle $S_{1}$ of $\gamma^{*} V$ defines a map

$$
\gamma_{1}: X \longrightarrow G(n+1,2)
$$

of $X$ into the Grassmannian of two planes in $\mathbb{C}^{n+1}$.

Now assume that $\gamma_{1}$ is an immersion. Then repeating the above argument we get a map

$$
\gamma_{2}: X \longrightarrow G(n+1,3)
$$

of $X$ into the Grassmannian of three planes in $\mathbb{C}^{n+1}$.

More generally, inductively we have a map

$$
\gamma_{i}: X \longrightarrow G(n+1, i+1),
$$

where $i \in[1, n-1]$, by assuming that $\gamma_{i-1}$ is an immersion. (See also [9, Section 1] for the details of the construction of the maps $\gamma_{i}$ described above.)

The condition that the map $\gamma$, together with each map $\gamma_{i}$, where $i \in[1, n-1]$, is an immersion, is equivalent to the condition that the map $\gamma$ is everywhere locally nondegenerate.

Now, we extend the above notion of everywhere locally nondegenerate map to the context of foliations, which we call transversely $\mathbb{C} \mathbb{P}^{n}$-structure.

Definition 2.3. A transversely $\mathbb{C P}^{n}$-structure on $\mathscr{F}$ is defined by giving a data $\left\{U_{i}, \phi_{i}\right\}_{i \in I}$ exactly as in Definition 2.1 satisfying conditions (1) and (2) and the following stronger version of (3): for every $i \in I$, there is an everywhere locally nondegenerate map

$$
\gamma_{i}: D_{i}:=\operatorname{image}\left(\phi_{i}\right) \longrightarrow \mathbb{C P}^{n}
$$

such that, for every pair $i, j \in I$, there is a commutative diagram of maps

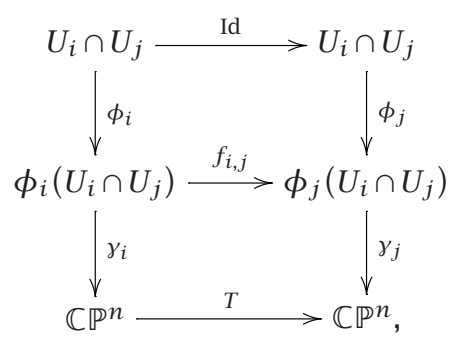

where $T$ is an automorphism of $\mathbb{C} \mathbb{P}^{n}$, that is, $T \in \mathrm{GL}(n+1, \mathbb{C})$. As before, two such data $\left\{U_{i}, \phi_{i}, \gamma_{i}\right\}_{i \in I}$ and $\left\{U_{i}, \phi_{i}, \gamma_{i}\right\}_{i \in J}$ are called equivalent if their union $\left\{U_{i}, \phi_{i}, \gamma_{i}\right\}_{i \in I \cup J}$ is 
also a data for a transversely $\mathbb{C P}^{n}$-structure. A transversely $\mathbb{C P}^{n}$-structure on $\mathscr{F}$ will mean an equivalence class of such data.

The above condition forces the map $f_{i, j}$ to be holomorphic. So, a transversely $\mathbb{C P}^{n}$ structure on $\mathscr{F}$ defines a transversely holomorphic structure on $\mathscr{F}$. If $\overline{\mathscr{F}}$ is a transversely holomorphic structure on $\mathscr{F}$, then a transversely $\mathbb{C P}^{n}$-structure on $\overline{\mathscr{F}}$ is a transversely $\mathbb{C P}^{n}$-structure on $\mathscr{F}$ such that the underlying transversely holomorphic structure coincides with $\overline{\mathscr{F}}$.

Note that, a transversely $\mathbb{C P}^{1}$-structure on $\mathscr{F}$ is by definition a transversely projective structure on $\mathscr{F}$.

We fix a transversely holomorphic structure $\overline{\mathscr{F}}$ on $\mathscr{F}$.

The normal bundle

$$
N:=\frac{T M}{\mathscr{F}}
$$

is a complex line bundle. Therefore, for every integer $k \in \mathbb{Z}$, we have a complex line bundle $N^{\otimes k}$ obtained by taking the $k$ th tensor power of the complex line bundle $N$. By $N^{\otimes-1}$ we mean the dual line bundle $N^{*}$.

Any such line bundle $N^{\otimes k}$ has a natural transversely holomorphic structure. This means that, there is a Dolbeault operator

$$
\bar{\partial}_{N^{\otimes k}}: N^{\otimes k} \longrightarrow N^{*} \otimes N^{\otimes k}=N^{\otimes k-1}
$$

satisfying the Leibniz identity. The operator $\bar{\partial}_{N^{\otimes k}}$ is simply the Dolbeault operator on the holomorphic tangent bundle $T_{\mathbb{C}}^{\otimes k}$ of the complex line $\mathbb{C}$ transported to $M$ using the projections $\phi_{i}$. It may be noted that, the condition in Definition 2.1(3) that every $f_{i, j}$ is holomorphic ensures that these locally defined operators patch compatibly to define the global differential operator $\bar{\partial}_{N^{\otimes k}}$.

Also, the line bundle $N$, and hence any $N^{\otimes k}$, has the Bott partial connection (see [8]). Recall that, the Lie bracket operation on the sheaf of sections of the tangent bundle $T M$ defines the Bott partial connection

$$
N \longrightarrow \mathscr{F}^{*} \otimes N
$$

along the foliation $\mathscr{F}$. The Jacobi identity for Lie bracket ensures that this partial connection is flat.

It is easy to see that both the complex structure of $N$ and the transversely holomorphic structure of $N$ are compatible with respect to the Bott partial connection. In other words, both the complex vector space structure of the fibers of $N$ and the Dolbeault operator $\bar{\partial}_{N}$ defined in (2.13) commute with the differential operator in (2.14) defining the Bott connection. Equivalently, parallel translation (for the Bott connection) along the leaves of the foliation $\bar{F}$ of holomorphic sections of $N$ remain holomorphic. Also, parallel translations for the Bott connection commute with multiplication by $\sqrt{-1}$ of the fibers of $N$.

The Bott partial connection on $N$ induces a flat partial connection on any $N^{\otimes k}$. All the above compatibility properties of the Bott connection on $N$ evidently remain valid for any $N^{\otimes k}$. 
Let $\mathscr{V}_{\overline{\mathscr{F}}}(k)$ denote the space of all globally defined smooth sections $s$ of the complex line bundle $N^{\otimes k}$ such that $s$ is transversely holomorphic for the transversely holomorphic foliation $\overline{\mathscr{F}}$ and it is flat with respect to the Bott partial connection for $\overline{\mathscr{F}}$. So $\mathscr{V}_{\overline{\mathscr{F}}}(k)$ is a complex vector space; it need not be of finite dimension. However, in the situation where $M$ is compact, it was proved by Duchamp and Kalka [4, Theorem 1.27, page 323], and also independently by Gómez-Mont [6, Theorem 1, page 169], that the dimension of $\mathscr{V}_{\bar{F}}(k)$ is finite.

Let $\mathscr{P}(\overline{\mathscr{F}})$ denote the space of all equivalence classes of transversely projective structures on the transversely holomorphic foliation $\overline{\mathscr{F}}$. Transversely projective structures were defined in Definition 2.2 and transversely projective structures on $\overline{\mathscr{F}}$ were defined in the paragraph following Definition 2.2. The space $\mathscr{P}(\overline{\mathscr{F}})$ may be empty.

The following theorem is the main result of this section.

THEOREM 2.4. There is a canonical bijective map from the space of all transversely $\mathbb{C P}^{n}$-structures on $\bar{F}$ and the Cartesian product

$$
\mathscr{P}(\overline{\mathscr{F}}) \times\left(\bigoplus_{k=3}^{n+1} \mathcal{V}_{\overline{\mathscr{F}}}(-k)\right) .
$$

In particular, a transversely $\mathbb{C P}^{n}$-structure gives a transversely projective structure on $\bar{F}_{\mathcal{F}}$ by simply taking the zero section in $\mathscr{V}_{\overline{\mathscr{F}}}(-k)$ for all $k \in[3, n+1]$.

The theorem will be proved after establishing a few lemmas. We start with the definition of jet bundles and differential operators.

Let $E$ be a holomorphic vector bundle on a Riemann surface $X$, and let $n$ be a positive integer. The $n$ th-order jet bundle of $E$, denoted by $J^{n}(E)$, is defined to be the following direct image on $X$ :

$$
J^{n}(E):=p_{1 *}\left(\frac{p_{2}^{*} E}{p_{2}^{*} E \otimes \mathcal{O}_{X \times X}(-(n+1) \Delta)}\right),
$$

where $p_{i}: X \times X \rightarrow X, i=1,2$, is the projection onto the $i$ th factor, and $\Delta$ is the diagonal divisor on $X \times X$. Therefore, for any $x \in X$, the fiber $J^{n}(E)_{x}$ is the space of all sections of $E$ over the $n$ th-order infinitesimal neighborhood of $x$.

Let $K_{X}$ denote the holomorphic cotangent bundle of $X$. There is a natural exact sequence

$$
0 \longrightarrow K_{X}^{\otimes n} \otimes E \longrightarrow J^{n}(E) \longrightarrow J^{n-1}(E) \longrightarrow 0
$$

constructed using the obvious inclusion of $\mathcal{O}_{X \times X}(-(n+1) \Delta)$ in $O_{X \times X}(-n \Delta)$. The inclusion map $K_{X}^{\otimes n} \otimes E \rightarrow J^{n}(E)$ is constructed by using the homomorphism

$$
K_{X}^{\otimes n} \longrightarrow J^{n}\left(O_{X}\right)
$$

which is defined at any $x \in X$ by sending $(d f)^{\otimes n}$, where $f$ is any holomorphic function with $f(x)=0$, to the jet of the function $f^{n} / n$ ! at $x$.

The sheaf of differential operators $\operatorname{Diff}_{X}^{n}(E, F)$ is defined to be $\operatorname{Hom}\left(J^{n}(E), F\right)$. The homomorphism

$$
\sigma: \operatorname{Diff}_{X}^{n}(E, F) \longrightarrow \operatorname{Hom}\left(K_{X}^{\otimes n} \otimes E, F\right)
$$


obtained by restricting a homomorphism from $J^{n}(E)$ to $F$ to the subsheaf $K_{X}^{\otimes n} \otimes E$ in (2.17), is known as the symbol map.

Let $X$ denote a simply connected open subset of $\mathbb{C P}^{1}$. Take a holomorphic map $\gamma: X \rightarrow \mathbb{C P}^{n}$. Let $\zeta$ denote the line bundle $\gamma^{*} \mathbb{O}_{\mathbb{C P}}(1)$ over $X$. In the notation of the exact sequence (2.4), the line bundle $\mathscr{O}_{\mathbb{C P} n}(1)$ is $S^{*}$. Pulling back the universal exact sequence (2.4) to $X$ and then taking the dual, we have

$$
0 \longrightarrow \gamma^{*} Q^{*} \longrightarrow W \stackrel{p}{\longrightarrow} \zeta \longrightarrow 0
$$

where $W$ is the trivial vector bundle of rank $n+1$ over $X$ with fiber $\left(\mathbb{C}^{n+1}\right)^{*}$. Of course, $\left(\mathbb{C}^{n+1}\right)^{*}=\mathbb{C}^{n+1}$.

The trivialization of $W$ induces a homomorphism

$$
\bar{p}: W \longrightarrow J^{n}(\zeta)
$$

which can be defined as follows: for any point $x \in X$ and vector $w \in W_{x}$ in the fiber, let $\bar{w}$ denote the unique flat section of $W$ such that $\bar{w}(x)=w$. Now, $\bar{p}(w)$ is the restriction of the section $p(\bar{w})$ of $\zeta$ to the $n$ th-order infinitesimal neighborhood of $x$. Recall that, the fiber $J^{n}(\zeta)_{x}$ is the space of sections of $\zeta$ over the $n$ th-order infinitesimal neighborhood of $x$.

LEMMA 2.5. The map $\gamma$ is everywhere locally nondegenerate if and only if the homomorphism $\bar{p}$ in (2.21) is an isomorphism.

Proof. This is a straightforward consequence of the condition of everywhere locally nondegeneracy. For some point $x \in X$, if $\bar{p}_{x}: W_{x} \rightarrow J^{n}(\zeta)_{x}$ is not an isomorphism, then take a nonzero vector $w$ in the kernel of $\bar{p}_{x}$, since $W_{x}=\left(\mathbb{C}^{n+1}\right)^{*}$, the vector $w$ defines a hyperplane $H$ in $\mathbb{C P}^{n}$. Clearly, $H$ contains $\gamma(x)$. The given condition $\bar{p}_{x}(w)=0$ can be seen to be equivalent to the condition that the order of contact of $H$ with $\gamma(X)$ at $\gamma(x)$ is at least $n$. In other words, $\gamma$ is degenerate at $x$.

Conversely, if $\gamma$ is degenerate at a point $x \in X$, take a hyperplane $H$ in $\mathbb{C P}^{n}$ containing $\gamma(x)$ such that the order of contact between $\gamma(X)$ and $H$ at $\gamma(x)$ is at least $n$. Let $w \in\left(\mathbb{C}^{n+1}\right)^{*}$ be a functional defining the hyperplane $H$. It is easy to see that $\bar{p}_{x}(w)=0$. This completes the proof.

Assume that $\gamma$ is everywhere locally nondegenerate. So the homomorphism $\bar{p}$ in (2.21) gives a trivialization of the jet bundle $J^{n}(\zeta)$. Now, from (2.17) it follows that $\bigwedge^{n+1} J^{n}(\zeta)$ is canonically isomorphic to $K_{X}^{n(n+1) / 2} \otimes \zeta^{n+1}$. The trivialization of $J^{n}(\zeta)$ induces a trivialization of $K_{X}^{n(n+1) / 2} \otimes \zeta^{n+1}$. Fix a square-root $\xi$ of the holomorphic tangent bundle $T_{X}$. In other words, $\xi$ is a holomorphic line bundle and an isomorphism between $T_{X}$ and $\xi^{\otimes 2}$ is chosen. The above trivialization of $K_{X}^{n(n+1) / 2} \otimes \zeta^{n+1}$ induces an isomorphism

$$
J^{i}\left(\zeta^{j}\right)=J^{i}\left(\xi^{n j}\right) \otimes\left(\xi^{n j}\right)^{*} \otimes \zeta^{j}
$$

for every $i$ and $j$. Indeed, this is an immediate consequence of the fact that $\zeta$ and $\xi^{n}$ differ by tensoring with a finite-order line bundle. By a finite-order line bundle we mean a line bundle some tensor power of which has a canonical trivialization. 
Consider the homomorphism

$$
\hat{p}: W \rightarrow J^{n+1}(\zeta)
$$

which sends any $w \in W_{x}$ to the restriction of the section $p(\bar{w})$ of $\zeta$ to the $(n+1)$ thorder infinitesimal neighborhood of $x$. Here $p$ as in (2.20) and $\bar{w}$ as in the definition of the map $\bar{p}$ in (2.21). From its definition it is immediate that the composition $f_{n} \circ \hat{p} \circ \bar{p}^{-1}$ is the identity map of $J^{n}(\zeta)$, where $f_{n}$ is the projection $J^{n+1}(\zeta) \rightarrow J^{n}(\zeta)$ defined in (2.17). In other words, $\hat{p} \circ \bar{p}^{-1}$ is a splitting of the jet sequence

$$
0 \longrightarrow K_{X}^{n+1} \otimes \zeta \longrightarrow J^{n+1}(\zeta) \longrightarrow J^{n}(\zeta) \longrightarrow 0
$$

defined in (2.17).

There is a unique homomorphism $J^{n+1}(\zeta) \rightarrow K_{X}^{n+1} \otimes \zeta$ satisfying the two conditions that its kernel is the image of $\hat{p} \circ \bar{p}^{-1}$ and the composition of the natural inclusion of $K_{X}^{n+1} \otimes \zeta$ in $J^{n+1}(\zeta)$ (as in (2.17)) with it is the identity map of $K_{X}^{n+1} \otimes \zeta$. By the earlier definition of differential operators given in terms of jet bundles, this homomorphism defines a differential operator

$$
D_{\gamma} \in H^{0}\left(X, \operatorname{Diff}_{X}^{n+1}\left(\zeta, K_{X}^{n+1} \otimes \zeta\right)\right) .
$$

Since $D_{\gamma}$ is defined by a splitting of a jet sequence, its symbol is the constant function 1 (the symbol of a differential operator is defined in (2.19)). Now, using (2.22), the differential operator $D_{\gamma}$ gives a differential operator

$$
D(\gamma) \in H^{0}\left(X, \operatorname{Diff}_{X}^{n+1}\left(\xi^{n}, \xi^{-n-2}\right)\right)
$$

of symbol 1.

It can be deduced from the definition of jet bundles that, for any holomorphic vector bundle $E$, there is a natural injective homomorphism $J^{i+j}(E) \rightarrow J^{i}\left(J^{j}(E)\right)$ for any $i, j \geq 0$. Therefore, we have a commutative diagram

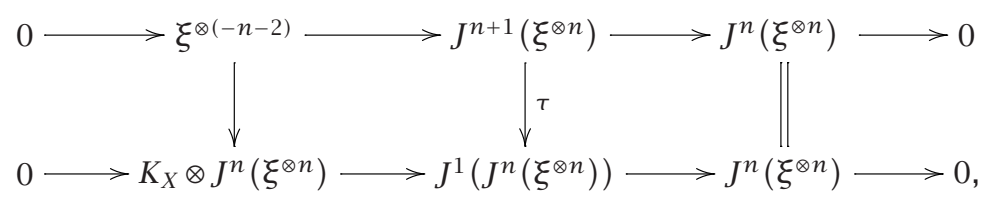

where the injective homomorphism $\tau$ is obtained from the above remark.

If

$$
f: J^{n}\left(\xi^{\otimes n}\right) \longrightarrow J^{n+1}\left(\xi^{\otimes n}\right)
$$

is a splitting of the top exact sequence in (2.27), then the composition $\tau \circ f$ defines a splitting of the bottom exact sequence in (2.27). But a splitting of the exact sequence

$$
0 \longrightarrow K_{X} \otimes E \longrightarrow J^{1}(E) \longrightarrow E \longrightarrow 0
$$

is a holomorphic connection on $E$ (see [1]). Furthermore, any holomorphic connection on a Riemann surface is flat. Therefore, $\tau \circ f$ defines a flat connection on $J^{n}\left(\xi^{\otimes n}\right)$. Let $\nabla^{f}$ denote this flat connection on $J^{n}\left(\xi^{\otimes n}\right)$ obtained from a splitting $f$. 
Since $X$ is simply connected, $\nabla^{f}$ gives a trivialization of $J^{n}\left(\xi^{\otimes n}\right)$. In other words, if we choose a point $z \in X$, using parallel translations, $J^{n}\left(\xi^{\otimes n}\right)$ gets identified with the trivial vector bundle over $X$ with $J^{n}\left(\xi^{\otimes n}\right)_{z}$ as the fiber.

Fix an isomorphism of the fiber $J^{n}\left(\xi^{\otimes n}\right)_{z}$ with $\mathbb{C}^{n+1}$. As before, let $W$ denote the trivial vector bundle over $X$ with $\mathbb{C}^{n+1}$ as the fiber. So we have $J^{n}\left(\xi^{\otimes n}\right)=W$.

For any point $y \in X$, consider the one-dimensional subspace $\left(\xi^{\otimes n} \otimes K_{X}^{n}\right)_{y}$ of the fiber $J^{n}\left(\xi^{\otimes n}\right)$ y given in (2.17). Let

$$
\gamma: X \rightarrow \mathbb{C P}^{n}
$$

denote the map that sends any point $y \in X$ to the line in $\mathbb{C}^{n+1}$ that corresponds to the line $\left(\xi^{\otimes n} \otimes K_{X}^{n}\right)_{y}$ by the isomorphism between the fibers $J^{n}\left(\xi^{\otimes n}\right)_{y}$ and $W_{y}$.

If we change the isomorphism between $J^{n}\left(\xi^{\otimes n}\right)_{z}$ and $\mathbb{C}^{n+1}$ by an automorphism $A \in \mathrm{GL}(n+1, \mathbb{C})$, then the map $\gamma$ is altered by the automorphism $A$ of $\mathbb{C} \mathbb{P}^{n}$.

LEMMA 2.6. Let $f: J^{n}\left(\xi^{\otimes n}\right) \rightarrow J^{n+1}\left(\xi^{\otimes n}\right)$ be a splitting of the top exact sequence in (2.27). Then the map $\gamma$ constructed in (2.30) from $f$ is everywhere locally nondegenerate.

Proof. The lemma follows from Lemma 2.5 and the fact that the connection $\nabla^{f}$, from which $\gamma$ is constructed, is given by a splitting $f$ (as in (2.28)). In [3], a different but equivalent formulation of the lemma can be found.

Two everywhere locally nondegenerate maps $f_{1}$ and $f_{2}$ of $\mathrm{X}$ into $\mathbb{C P}^{n}$ are called equivalent if there is an automorphism $A \in \operatorname{Aut}\left(\mathbb{C P}^{n}\right)=\operatorname{PGL}(n+1, \mathbb{C})$ such that $A$ 。 $f_{1}=f_{2}$.

Let A denote the space of all equivalence classes of everywhere locally nondegenerate maps of $X$ into $\mathbb{C} \mathbb{P}^{n}$.

Take a differential operator $D \in H^{0}\left(X, \operatorname{Diff}_{X}^{n+1}\left(\xi^{n}, \xi^{-n-2}\right)\right)$ of symbol 1 . Since the symbol of $D$ is 1 , it gives a splitting of the top exact sequence in (2.27). Denoting this splitting $J^{n}\left(\xi^{\otimes n}\right) \rightarrow J^{n+1}\left(\xi^{\otimes n}\right)$ by $\bar{D}$, consider $\tau \circ \bar{D}$, which, as we already noted, is a flat connection on $J^{n}\left(\xi^{\otimes n}\right)$. It may be noted that since $\xi^{\otimes 2}=T_{X}$, the line bundle $\bigwedge^{n+1} J^{n}\left(\xi^{\otimes n}\right)$ is canonically trivialized.

Let $\Re$ denote the space of global differential operators

$$
D \in H^{0}\left(X, \operatorname{Diff}_{X}^{n+1}\left(\xi^{n}, \xi^{-n-2}\right)\right)
$$

of symbol 1 and satisfying the condition that the connection on $\wedge^{n+1} J^{n}\left(\xi^{\otimes n}\right)$ induced by the connection $\tau \circ \bar{D}$ on $J^{n}\left(\xi^{\otimes n}\right)$ preserves the trivialization of $\bigwedge^{n+1} J^{n}\left(\xi^{\otimes n}\right)$.

From the construction of the differential operator $D(\gamma)$ in (2.26) it follows that $D(\gamma) \in \mathscr{B}$.

Let

$$
F: \mathscr{A} \longrightarrow \mathscr{B}
$$

be the map that sends any everywhere locally nondegenerate map $\gamma$ to the differential operator $D(\gamma)$ constructed in (2.26).

As above, for a differential operator $D \in \mathscr{B}$, the corresponding splitting is denoted by $\bar{D}$. Let

$$
G: \mathscr{B} \rightarrow A
$$


be the map that sends any operator $D$ to the map $\gamma$ constructed in (2.30) using the splitting $f=\bar{D}$ as in (2.28).

LEMMA 2.7. The map $F$ defined in (2.32) is one-to-one and onto.

Proof. In fact, unraveling the definitions of the maps $F$ and $G$, defined in (2.32) and (2.33), respectively, yields that they are inverses of each other. We omit the details; it can be found in [3].

Let $\mathscr{P}(X)$ denote the space of all projective structures on the Riemann surface $X$. It is known that $\mathscr{P}(X)$ is an affine space for the space of quadratic differentials, namely, $H^{0}\left(X, K_{X}^{2}\right)$ (see [7]).

LEMмA 2.8. There is a natural bijective map between $\mathscr{B}$ and the Cartesian product

$$
\mathscr{P}(X) \times\left(\bigoplus_{i=3}^{n+1} H^{0}\left(X, K_{X}^{\otimes i}\right)\right)
$$

if $n \geq 2$. If $n=1$, then $\mathscr{B}$ is in bijective correspondence with $\mathscr{P}(X)$.

Proof. The key input in the proof is [2, Theorem 6.3, page 19]. Now we recall its statement.

Let $Y$ be a Riemann surface equipped with a projective structure. Let $k, l \in \mathbb{Z}$ and let $n \in \mathbb{N}$ be such that $k \notin[-n+1,0]$ and $l-k-j \notin\{0,1\}$ for any integer $j \in[1, n]$. Then,

$$
H^{0}\left(Y, \operatorname{Diff}_{Y}^{n}\left(\mathscr{L}^{k}, \mathscr{L}^{l}\right)\right)=\bigoplus_{i=0}^{n} H^{0}\left(Y, \mathscr{L}^{l-k-2 n+2 i}\right),
$$

where $\mathscr{L}$ is the square-root of the canonical bundle defined by the projective structure.

A clarification of the above statement is needed. In [2], a projective structure means an $\operatorname{SL}(2, \mathbb{C})$ structure. But here projective structure means a PGL(2, $\mathbb{C})$ structure. But we know that a PGL $(2, \mathbb{C})$ structure on a Riemann surface always lifts to an $\operatorname{SL}(2, \mathbb{C})$ structure [7]. Furthermore, the space of such lifts is in bijective correspondence with the space of theta-characteristics (square-root of the holomorphic cotangent bundle) of $Y$.

Therefore, given a PGL $(2, \mathbb{C})$ structure $P$ on $X$, the pair $(P, \xi)$ determines a unique $\operatorname{SL}(2, \mathbb{C})$ structure.

Now, set $k=-n$ and $l=n+2$ in (2.35). This yields an isomorphism

$$
F: H^{0}\left(X, \operatorname{Diff}_{X}^{n+1}\left(\xi^{n}, \xi^{-n-2}\right)\right) \longrightarrow \bigoplus_{i=0}^{n} H^{0}\left(X, K_{X}^{\otimes i}\right) .
$$

For any $D \in H^{0}\left(X, \operatorname{Diff}_{X}^{n+1}\left(\xi^{n}, \xi^{-n-2}\right)\right)$, the component of $F(D)$ in

$$
H^{0}\left(X, K_{X}^{\otimes 0}\right)=H^{0}\left(X, \mathcal{O}_{X}\right)
$$

is the symbol of $D$. Furthermore, the condition in the definition of $\mathscr{B}$ that, the connection on $\bigwedge^{n+1} J^{n}\left(\xi^{\otimes n}\right)$ induced by the connection $\tau \circ \bar{D}$ on $J^{n}\left(\xi^{\otimes n}\right)$ preserves the trivialization of $\bigwedge^{n+1} J^{n}\left(\xi^{\otimes n}\right)$, is actually equivalent to the condition that the component of $F(D)$ in $H^{0}\left(X, K_{X}\right)$ vanishes (see [3]). Therefore, using $F$, the space $\mathscr{B}$ gets 
identified with the direct sum

$$
\bigoplus_{i=2}^{n+1} H^{0}\left(X, K_{X}^{\otimes i}\right)
$$

if $X$ is equipped with a projective structure.

Using the fact that the space of projective structures on $X$, namely $\mathscr{P}(X)$, is an affine space for $H^{0}\left(X, K_{X}^{2}\right)$, it is easy to deduce that given any

$$
D \in H^{0}\left(X, \operatorname{Diff}_{X}^{n+1}\left(\xi^{n}, \xi^{-n-2}\right)\right),
$$

there is a unique projective structure $P \in \mathscr{P}(X)$ such that, for the map $F$ in (2.36) corresponding to $P$, the component of $F(D)$ in $H^{0}\left(X, K_{X}^{\otimes 2}\right)$ vanishes identically. Let $\overline{F(D)}$ denote the projection of $F(D)$ in $\bigoplus_{i=3}^{n+1} H^{0}\left(X, K_{X}^{\otimes i}\right) ; F$ corresponds to this unique projective structure. Now, we have a bijective map

$$
\bar{F}: \mathscr{B} \longrightarrow \mathscr{P}(X) \times\left(\bigoplus_{i=3}^{n+1} H^{0}\left(X, K_{X}^{\otimes i}\right)\right),
$$

that sends any $D$ to the pair $(P, \overline{F(D)})$ constructed above. (See [3, Section 4] for the details.)

If $n=1$, then using [2, Theorem 6.3] and the fact that $\mathscr{P}(X)$ is an affine space for $H^{0}\left(X, K_{X}^{\otimes 2}\right)$, it follows immediately that $\mathscr{B}=\mathscr{P}(X)$. This completes the proof of the lemma.

For the first part of the proof of Lemma 2.8, we should have directly used [2, Corollary 6.6] instead of deriving it using [2, Theorem 6.3]. Unfortunately, in the statement of [2, Corollary 6.6], the word "compact" is used which technically makes it useless for our purpose. But, of course, compactness is not used in the proof of [2, Corollary 6.6]. When [2, 3] were written, we had primarily compact Riemann surfaces in mind.

Combining Lemmas 2.7 and 2.8, we have the following corollary.

COROLLARY 2.9. There is a natural bijective map

$$
\Gamma: \mathscr{A} \longrightarrow \mathscr{P}(X) \times\left(\bigoplus_{i=3}^{n+1} H^{0}\left(X, K_{X}^{\otimes i}\right)\right)
$$

for $n \geq 2$. If $n=1$ then $\mathscr{A}$ is in bijective correspondence with $\mathscr{P}(X)$.

When $X$ is a compact Riemann surface, the above corollary is [3, Theorem 5.5]. Again since "compactness" condition is thrown in [3] indiscriminately, a vast part of it is technically useless for our present purpose. Nevertheless, the ideas of [3] have been borrowed here.

Let $Y \subset X$ be a simply connected open subset. Let $\mathscr{A}_{Y}$ denote the space of all equivalence classes of everywhere locally nondegenerate maps of $Y$ into $\mathbb{C} \mathbb{P}^{n}$. In other words, $\mathscr{A}_{Y}$ is obtained by substituting $Y$ in place of $X$ in the definition of $\mathscr{A}$. The space of all projective structures on $Y$ is denoted by $\mathscr{P}(Y)$. 
The restriction of $\xi$ to $Y$ defines a square-root of the tangent bundle $T_{Y}$. There is a natural restriction map $\mathscr{P}(X) \rightarrow \mathscr{P}(Y)$ and also there are homomorphisms

$$
H^{0}\left(X, K_{X}^{\otimes i}\right) \longrightarrow H^{0}\left(Y, K_{Y}^{\otimes i}\right)
$$

for every $i \in \mathbb{Z}$ defined by restriction of sections. Similarly, we have a map $A_{A} \rightarrow \mathscr{A}_{Y}$, which sends a map $\gamma$ of $X$ to $\mathbb{C P}^{n}$ to the restriction of $\gamma$ to $Y$.

Let

$$
\Gamma_{Y}: \mathscr{A}_{Y} \longrightarrow \mathscr{P}(Y) \times\left(\bigoplus_{i=3}^{n+1} H^{0}\left(Y, K_{Y}^{\otimes i}\right)\right)
$$

be the isomorphism for $Y$ obtained in Corollary 2.9. The map $\Gamma$ in Corollary 2.9 has the property that the following diagram commutes:

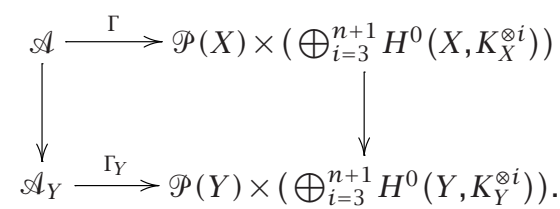

The vertical maps are defined by restriction. The commutativity of this diagram is indeed easy to see from the construction of $\Gamma$.

Now that we have Corollary 2.9 and (2.44), we are ready to prove Theorem 2.4.

Proof OF Theorem 2.4. Assume that $n \geq 2$, since the theorem is obvious in the case of $n=1$.

Suppose we are given a transversely $\mathbb{C}^{n}$-structure, as defined in Definition 2.3. We assume that all the subsets $D_{i}:=\operatorname{image}\left(\phi_{i}\right)$ of $\mathbb{C}$ in Definition 2.1 are simply connected. Clearly, this is a harmless assumption.

Consider a triplet $\left(U_{i}, \phi_{i}, \gamma_{i}\right)$ as in Definition 2.3. Now, using the map $\Gamma$ in Corollary 2.9 , from the everywhere locally nondegenerate map $\gamma_{i}$ we have a projective structure on $D_{i}=\operatorname{image}\left(\phi_{i}\right)$ together with a holomorphic section of $T_{D_{i}}^{\otimes-l}$ for all $l \in[3, n+1]$. This projective structure on $D_{i}$ is denoted by $\mathscr{P}_{i}$, and the holomorphic section of $T_{D_{i}}^{\otimes-l}$ obtained above is denoted by $\omega_{i}^{l}$. The projective structure $\mathscr{P}_{i}$ induces a transversely projective structure on the open subset $U_{i}$ of $M$. We denote this transversely projective structure on $U_{i}$ by $\overline{\mathscr{P}}_{i}$. The pullback, using the map $\phi_{i}$, of the holomorphic section $\omega_{i}^{l}$ of $T_{D_{i}}^{\otimes-l}$ defines a section of $N^{\otimes-l}$ over $U_{i}$. This section of $N^{\otimes-l}$ over $U_{i}$ is denoted by $\bar{\omega}_{i}^{l}$. Since $\omega_{i}^{l}$ is holomorphic, we have the section $\bar{\omega}_{i}^{l}$ over $U_{i}$ to be transversely holomorphic. Furthermore, $\bar{\omega}_{i}^{l}$ is obviously flat with respect to the Bott partial connection. The proof of the theorem is completed by showing that all these locally defined transversely projective structures $\overline{\mathscr{P}}_{i}$ (resp., transversely holomorphic flat sections $\bar{\omega}_{i}^{l}$ ) patch compatibly to define globally on $M$ a transversely projective structure (resp., transversely holomorphic flat section of $N^{\otimes-l}$ ).

If we take another triplet $\left(U_{j}, \phi_{j}, \gamma_{j}\right), j \in I$, as in Definition 2.3, then the two projective structures on $D_{i} \cap D_{j}$, namely $\mathscr{P}_{i}$ and $\mathscr{P}_{j}$, coincide. This is an immediate consequence of the commutativity of the diagram (2.44). Therefore, we have a projective 
structure on the union $D_{i} \cup D_{j}$, and hence the two transversely projective structures, namely $\overline{\mathscr{P}}_{i}$ and $\overline{\mathscr{P}}_{j}$, coincide over $U_{i} \cap U_{j}$. Consequently, the transversely projective structures $\left\{\overline{\mathscr{P}}_{i}\right\}_{i \in I}$ patch together compatibly to define a transversely projective structure on $\overline{\mathscr{F}}$. Similarly, from the commutativity of the diagram (2.44), it follows that the two sections $\bar{\omega}_{i}^{l}$ and $\bar{\omega}_{j}^{l}$ coincide over $U_{i} \cap U_{j}$. In other words, these local sections $\bar{\omega}_{i}^{l}$ of $N^{\otimes-l}$ patch together to give an element of $\mathscr{V}_{\overline{\mathscr{F}}}(-l)$. This completes the proof of the theorem.

Theorem 2.4 can be considered as a generalization of [10, Theorem 6.1].

\section{REFERENCES}

[1] M. F. Atiyah, Complex analytic connections in fibre bundles, Trans. Amer. Math. Soc. 85 (1957), 181-207.

[2] I. Biswas, Differential operators on complex manifolds with a flat projective structure, J. Math. Pures Appl. (9) 78 (1999), 1-26.

[3] __ Invariants for a class of equivariant immersions of the universal cover of a compact Riemann surface into a projective space, J. Math. Pures Appl. (9) 79 (2000), 1-20.

[4] T. Duchamp and M. Kalka, Deformation theory for holomorphic foliations, J. Differential Geom. 14 (1979), 317-337.

[5] C. Godbillon, Feuilletages. Études Géométriques [Foliations. Geometric Studies], Progress in Mathematics, vol. 98, Birkhäuser Verlag, Basel, 1991 (French).

[6] X. Gómez-Mont, Transversal holomorphic structures, J. Differential Geom. 15 (1980), 161185.

[7] R. C. Gunning, Lectures on Riemann Surfaces, Princeton Mathematical Notes, Princeton University Press, New Jersey, 1966.

[8] H. B. Lawson Jr., The Quantitative Theory of Foliations, vol. 27, American Mathematical Society, Rhode Island, 1977.

[9] D. Perkinson, Curves in Grassmannians, Trans. Amer. Math. Soc. 347 (1995), 3179-3246.

[10] B. A. Scárdua, Transversely affine and transversely projective holomorphic foliations, Ann. Sci. École Norm. Sup. (4) 30 (1997), 169-204.

INDRANIL BISWAS: SCHOOL OF MATHEMATICS, TATA INSTITUTE OF FUNDAMENTAL RESEARCH, HOMI BHABHA ROAD, BOMBAY 400005, INDIA

E-mail address: indrani1@math.tifr.res. in 


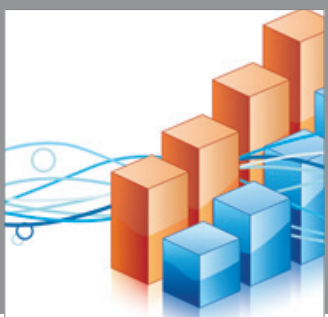

Advances in

Operations Research

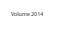

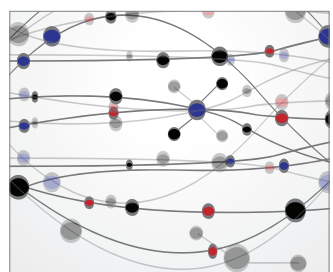

\section{The Scientific} World Journal
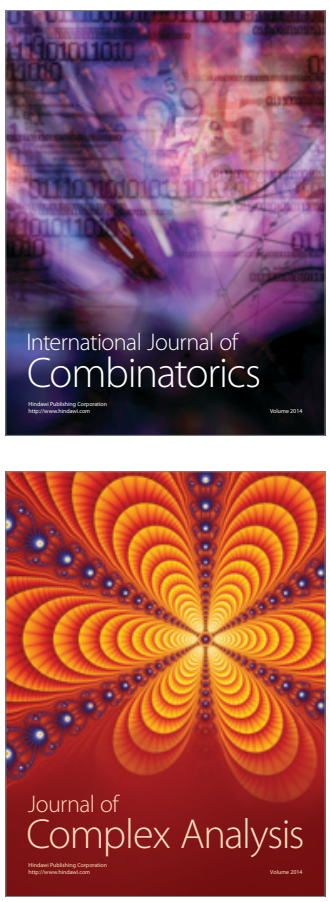

International Journal of

Mathematics and

Mathematical

Sciences
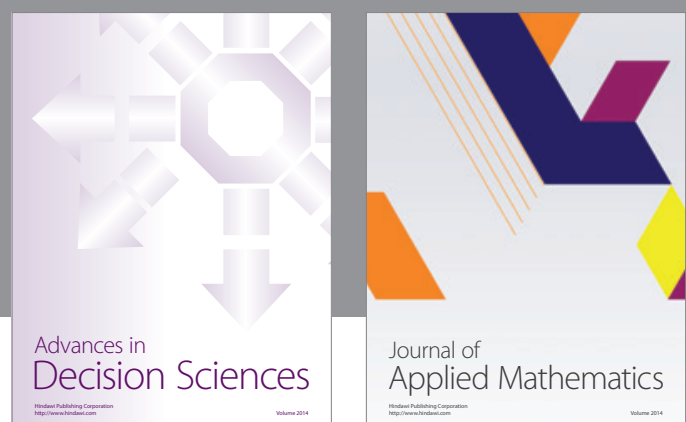

Journal of

Applied Mathematics
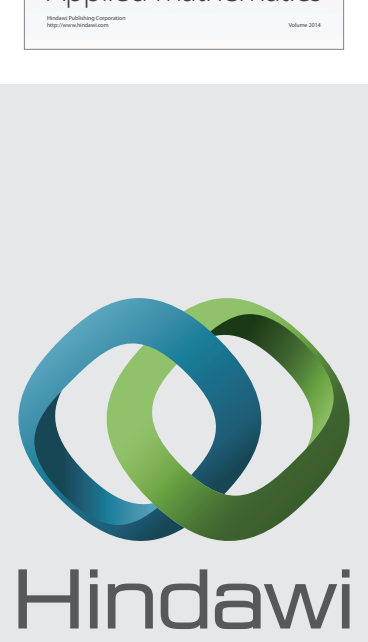

Submit your manuscripts at http://www.hindawi.com
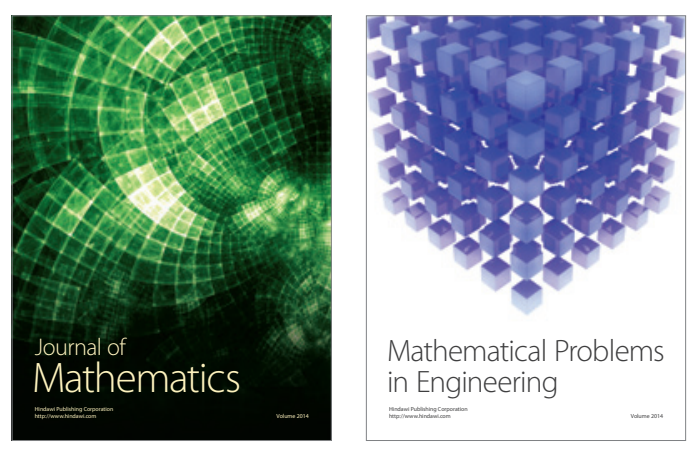

Mathematical Problems in Engineering
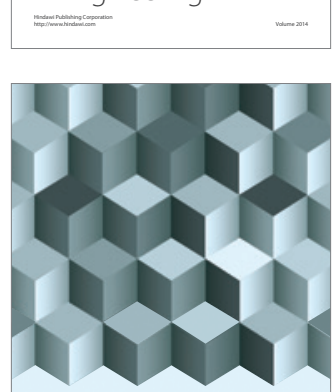

Journal of

Function Spaces
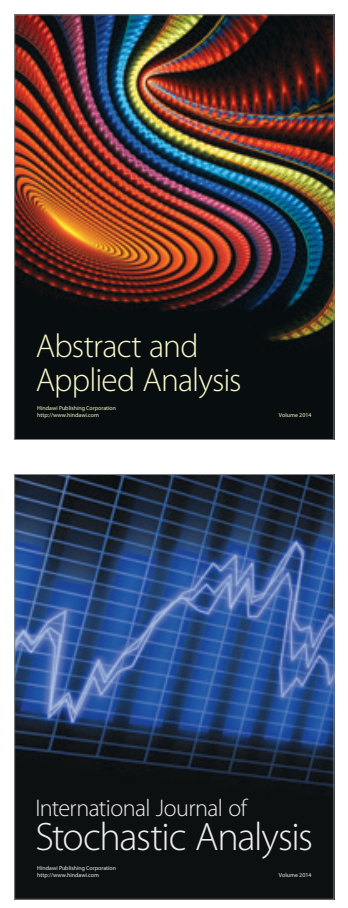

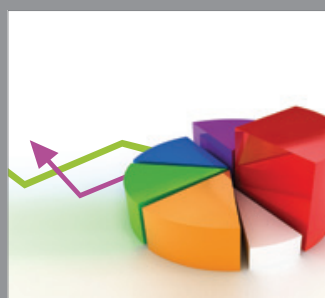

ournal of

Probability and Statistics

Promensencen
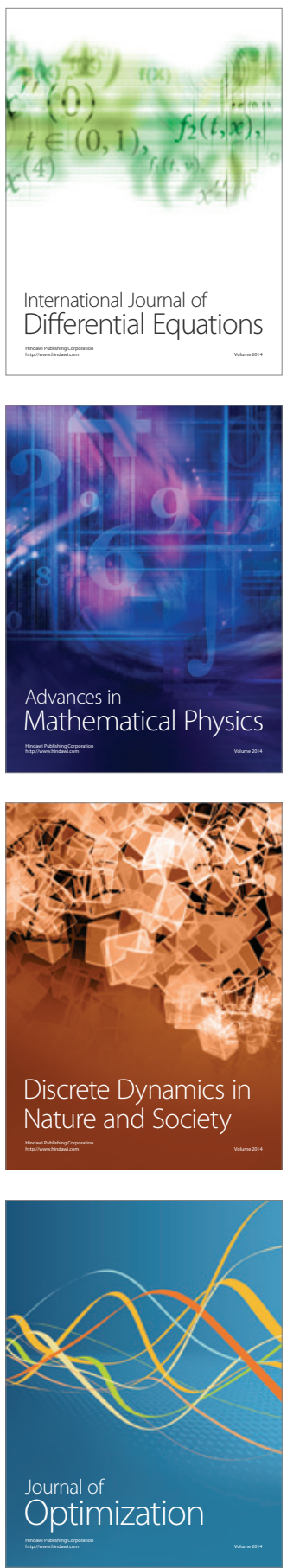